\title{
Array CGH as a first line diagnostic test in place of karyotyping for postnatal referrals - results from four years' clinical application for over 8,700 patients
}

Joo Wook Ahn ${ }^{1 *}$, Susan Bint ${ }^{2}$, Anne Bergbaum², Kathy Mann ${ }^{2}$, Richard P Hall ${ }^{2}$ and Caroline Mackie Ogilvie

\begin{abstract}
Background: Array CGH is widely used in cytogenetics centres for postnatal constitutional genome analysis, and is now recommended as a first line test in place of G-banded chromosome analysis. At our centre, first line testing by oligonucleotide array CGH for all constitutional referrals for genome imbalance has been in place since June 2008, using a patient vs patient hybridisation strategy to minimise costs.

Findings: Out of a total of 13,412 patients tested with array CGH, 8,794 (66\%) had array CGH as the first line test. Referral indications for this first line group ranged from neonatal congenital anomalies through to adult neurodisabilities; $25 \%$ of these patients had CNVs either in known pathogenic regions or in other regions where imbalances have not been reported in the normal population. Of these CNVs, $46 \%$ were deletions or nullisomy, $53 \%$ were duplications or triplications, and mosaic imbalances made up the remainder; $87 \%$ were $<5 \mathrm{Mb}$ and would likely not be detected by G-banded chromosome analysis. For cases with completed inheritance studies, 20\% of imbalances were de novo.

Conclusions: Array CGH is a robust and cost-effective alternative to traditional cytogenetic methodology; it provides a higher diagnostic detection rate than G-banded chromosome analysis, and adds to the sum of information and understanding of the role of genomic imbalance in disease. Use of novel hybridisation strategies can reduce costs, allowing more widespread testing.
\end{abstract}

Keywords: Array CGH, First line testing, G-banded karyotype analysis, CNV

\section{Background}

Array CGH $(\mathrm{aCGH})$ has a much higher resolution than G-banded chromosome analysis and most cytogenetic departments are now using this approach either as an adjunct to G-banded chromosome analysis, or as a firstline test for selected patient groups [1,2]. The implementation of oligonucleotide aCGH at our centre has been described in a previous paper [3]; this service has been offered since May 2008 using a patient vs patient (phenotype mismatched) hybridisation strategy to minimise costs, an important consideration in a state-funded health

\footnotetext{
* Correspondence: joowook.ahn@nhs.net

'Cytogenetics Department, Guy's and St Thomas' NHS Foundation Trust, London SE1 9RT, UK

Full list of author information is available at the end of the article
}

service; first line testing by aCGH for all constitutional referrals for genome imbalance has been in place since September 2008. We have now tested a total of 13,412 samples; here, we report on our findings for all samples where aCGH was used as a first line test $(\mathrm{n}=8,794)$.

\section{Patients}

Patients were referred from paediatric, neonatal and adult disability populations within our NHS regional area (population $\sim 6 \mathrm{M}$ ), and from other centres both in the UK and abroad. The median age for the first line testing group was 4 years (range: newborn - 78yrs); referrals were for developmental delay, more specific neurodisability (autism, ADHD, etc.), congenital abnormalities, dysmorphism, or other specific phenotypes (eg café au lait patches). 


\section{Methodology}

Genomic DNA extracted from peripheral blood or saliva, or DNA provided by external laboratories, was processed as previously described [3]. Briefly, samples were co-hybridised with other samples mismatched for phenotype and matched for sex (thus halving consumable costs compared with patient vs control). Agilent 4x44k oligonucleotide array platform AMADID 017457 was initially used, replaced in 2010 by an $8 \times 60 \mathrm{k}$ platform (AMADID 028469) which included additional probes in regions of clinical interest, and in the pseudoautosomal regions. Analysis was performed using Agilent algorithm ADM-2, threshold 6 and a 3-probe minimum aberration call; a further analysis using ADM-1 was carried out to maximise detection of mosaicism [4]. Imbalances of regions represented in the Database of Genomic Variants [5] in at least three non-BAC based studies were classified benign, and recorded but not reported. All samples with other imbalances were re-tested using G-banded karyotyping, QF-PCR, FISH, custom MLPA [6] or a repeat array.

\section{Turn-around times and success rates}

The average reporting time for first-line tests over the entire period was 21 days from receipt of sample. Hybridisations that did not meet QC metric thresholds (DLRS, \% non-uniform outliers, signal intensity and signal to noise) were repeated. Occasionally, it was not possible to complete testing, usually due to DNA degradation. The overall success rate was $99 \%$.

\section{Imbalances detected}

Tables 1 and 2 show the imbalances detected in 8,794 patients tested by first line aCGH. All individuals with features suggestive of Down, Edwards, Patau or Turner syndrome were first tested with QF-PCR [7] and if positive did not proceed to aCGH; hence the prevalence of these syndromes may appear lower in this patient group than in other reports. 25\% of patients had CNVs either in known pathogenic regions or in other regions where imbalances have not been reported in the normal population. Of these CNVs, 46\% were deletions or nullisomy, $53 \%$ were duplications or triplications, and mosaic imbalances made up the remainder; $87 \%$ were $<5 \mathrm{Mb}$ and would likely not be detected by traditional karyotyping. For cases with completed inheritance studies, 20\% of imbalances were de novo.

The most common findings in our dataset were imbalance in the 22q11.2 deletion syndrome region (OMIM $188400,192430,608363 ; 83$ cases), and deletion or duplication of the $16 \mathrm{p} 11.2$ autism susceptibility locus (OMIM 611913, 614671; 60 cases). Size of imbalances ranged from $<25 \mathrm{~kb}$ to whole chromosomes, with most pathogenic, syndromic and susceptibility locus imbalances being submicroscopic, whereas "private" imbalances ranged from $<25 \mathrm{~kb}$ to $105 \mathrm{Mb},(69 \%<5 \mathrm{Mb})$. All these diagnostic findings can be viewed on the UCSC Genome Browser via http://bbgre.org.

\section{Interpretation}

The size of imbalances with potential clinical significance generally correlated with severity of phenotype, although there were exceptions. For instance, a $\sim 7 \mathrm{Mb}$ duplication (4p15.2p15.1(23,365,794-30,530,905)x3) was found in two siblings, only one of whom had a clinical phenotype. It therefore seems unlikely that the duplication alone was causative in the affected sibling. Towards the other end of the size scale, a deletion of $157 \mathrm{~kb}$ that included part of SALL1 and no other genes was found in an infant with microcephaly, ear tags and imperforate anus; SALL1 mutation is associated with Townes-Brocks syndrome (OMIM 107480), consistent with the referral indication. These examples demonstrate the potential pitfalls of using arbitrary size cut-offs and the need for careful consideration of gene content of unbalanced regions when interpreting aCGH data.

\section{Inheritance studies}

We were only able to complete inheritance studies for $50 \%$ of patients with imbalances; $20 \%$ had imbalances that had arisen de novo. Inheritance patterns may be considered key to clinical interpretation of aCGH findings; however, it has become clear that simple rules cannot be used [8]. For instance, of our de novo findings, $8 \%(21 / 226)$ did not appear to be associated with the patient's clinical features; some included no genes or regulatory elements and were therefore unlikely to be clinically significant. Detection of benign de novo CNVs is unsurprising considering estimates for the de novo $\mathrm{CNV}$ mutation rate in the normal population [9]. Conversely, at least $20 \%$ of inherited imbalances represented susceptibility loci, and were therefore considered to be clinically significant; the clinical status of the carrier parent was generally not known. Penetrance of phenotype associated with these susceptibility loci is often variable; for instance, the $15 \mathrm{q} 13.3$ deletion syndrome imbalance has been reported with different clinical presentations within the same family [10].

\section{Structural information}

aCGH does not give information on the location in the genome of, for instance, duplicated regions, or on the structure of chromosomes. However, patterns of imbalance can be used to deduce this information in some cases; for instance, terminal deletion of one chromosome with duplication of terminal material of another chromosome is indicative of a derivative chromosome, and Gbanded chromosome analysis and/or FISH for the parents 
Table 1 Summary of findings from first line array CGH testing, June 2008 - Sept 2012

\begin{tabular}{|c|c|c|}
\hline & $\mathrm{n}$ & $\%$ \\
\hline TOTAL FIRST LINE PATIENTS & 8,794 & \\
\hline Abnormal & 2,218 & $25 \%$ (of total patients) \\
\hline Normal & 6,576 & \\
\hline Completed inheritance studies & 1,111 & $50 \%$ (of abnormals) \\
\hline De novo & 226 & $20 \%$ (of completed inheritance) \\
\hline Inherited & 885 & $80 \%$ (of completed inheritance) \\
\hline TOTAL IMBALANCES \# & 2,596 & \\
\hline Deletions / nullisomy (all chromosomes) & 1,182 & $46 \%$ (of all imbalances) \\
\hline Deletions (autosomes) & 1,102 & $42 \%$ (of all imbalances) \\
\hline Nullisomy (autosomes) & 8 & $<1 \%$ (of all imbalances) \\
\hline Deletions / nullisomy (sex chromosomes) & 72 & $3 \%$ (of all imbalances) \\
\hline Duplications (all chromosomes) & 1,240 & $48 \%$ (of all imbalances) \\
\hline Duplications (autosomes) & 951 & $37 \%$ (of all imbalances) \\
\hline Duplications (sex chromosomes) & 289 & $11 \%$ (of all imbalances) \\
\hline Triplications (all chromosomes) & 132 & $5 \%$ (of all imbalances) \\
\hline Triplications (autosomes) & 120 & $5 \%$ (of all imbalances) \\
\hline Triplications (sex chromosomes) & 12 & $<1 \%$ (of all imbalances) \\
\hline Amplifications & $1^{*}$ & $<1 \%$ (of all imbalances) \\
\hline Mosaics (all chromosomes) & 41 & $2 \%$ (of all imbalances) \\
\hline$x 0 \sim 1$ & 5 & $<1 \%$ (of all imbalances) \\
\hline $\mathrm{x} 1 \sim 2$ & 19 & $1 \%$ (of all imbalances) \\
\hline$x 1 \sim 3$ & 2 & $<1 \%$ (of all imbalances) \\
\hline$x 2 \sim 3$ & 13 & $1 \%$ (of all imbalances) \\
\hline$\times 2 \sim 4$ & 2 & $<1 \%$ (of all imbalances) \\
\hline Whole chromosome & 79 & $3 \%$ (of all imbalances) \\
\hline Whole chromosome mosaic & 19 & $1 \%$ (of all imbalances) \\
\hline Reduced copy number $>=5 \mathrm{Mb}$ & 74 & $3 \%$ (of all imbalances) \\
\hline Reduced copy number $<5 \mathrm{Mb}$ & 1,108 & 43\% (of all imbalances) \\
\hline Increased copy number $>=5 \mathrm{Mb}$ & 86 & $3 \%$ (of all imbalances) \\
\hline Increased copy number $<5 \mathrm{Mb}$ & 1,153 & $44 \%$ (of all imbalances) \\
\hline PATHOGENIC IMBALANCES \# & 868 & $33 \%$ (of all imbalances) \\
\hline Whole chromosome & 79 & 9\% (of pathogenic imbalances) \\
\hline Syndromic imbalances & 225 & $26 \%$ (of pathogenic imbalances) \\
\hline Susceptibility loci & 205 & $24 \%$ (of pathogenic imbalances) \\
\hline Other pathogenic (private mutations) & 359 & $41 \%$ (of pathogenic imbalances) \\
\hline
\end{tabular}

\# Patients may carry more than one pathogenic imbalance.

* This patient carried 5 copies in total of a region of chromosome 7.

is recommended in these cases. "Inv dup dels" can also be deduced (e.g. 5p15.33p14.1(148,243-27,385,955)x1,5p14.1p13.3 $(27,463,381-31,329,932) x 3)$, as can ring chromosomes (e.g. 18p11.32p11.21(170,229-14,918,854)x1 2,18q22.1q23(61,430, 694-76,083,117)x1 2), supernumerary ring chromosomes (8p12q11.1(35,817,703-47,655,281)x2 3) and inversion recombinants (e.g. 5p15.33p15.2(148,243-13,743,977)x1,
$5 q 35.2 q 35.3(172,591,725-180,617,107) \times 3)$. When aCGH is used as the first line test, cultured material from patients may not be available for immediate confirmation of any suspected structural rearrangements by karyotyping or FISH. The correct interpretation of array findings is therefore critical, as this will inform which follow-up studies are most appropriate. 
Table 2 Established genomic disorders detected

\begin{tabular}{|c|c|c|c|c|c|}
\hline OMIM & Syndrome & $\mathbf{n}$ & del $(x 1)$ & dup $(x 3)$ & $\operatorname{trp}(x 4)$ \\
\hline 607872 & $1 p 36$ & 2 & 2 & - & - \\
\hline $612474 / 612475$ & $1 \mathrm{q} 21.1^{\$}$ & 42 & 20 & 22 & - \\
\hline 600430 & $2 q 37$ & 4 & 4 & - & - \\
\hline $609425 / 611936$ & $3 q 29$ & 8 & 2 & 6 & - \\
\hline 194190 & Wolf-Hirschhorn & 2 & 2 & - & - \\
\hline 123450 & Cri du Chat & 3 & 3 & - & - \\
\hline 175100 & Familial Adenomatous Polyposis 1 & 1 & 1 & - & - \\
\hline 117550 & Sotos & 2 & 2 & - & - \\
\hline 194050/609757 & Williams-Beuren & 14 & 6 & 7 & 1 \\
\hline 183600 & Split-Hand/Foot Malformation 1 & 2 & 2 & - & - \\
\hline 610253 & Kleefstra & 4 & 4 & - & - \\
\hline 194072 & WAGR & 1 & 1 & - & - \\
\hline $176270 / 105830$ & Prader-Willi/Angelman & 19 & 10 & 7 & 2 \\
\hline 612001 & $15 q 13.3^{\$}$ & 26 & 26 & - & - \\
\hline $613406 / 613406$ & $15 q 24$ & 2 & 2 & - & - \\
\hline * & $15 q 26$ & 1 & 1 & - & - \\
\hline 141750 & ATR-16 & 2 & 2 & - & - \\
\hline ** & $16 p 13.11^{\$}$ & 45 & 13 & 32 & - \\
\hline 136570 & $16 \mathrm{p} 12.1^{\$}$ & 24 & 24 & - & - \\
\hline 613444 & Distal $16 p 11.2^{\$}$ & 8 & 8 & - & - \\
\hline 611913 & Proximal $16 \mathrm{p} 11.2^{\$}$ & 60 & 35 & 24 & 1 \\
\hline 247200 & Miller-Dieker & 9 & $4 \wedge$ & $5 \wedge$ & - \\
\hline $118220 / 162500$ & Charcot-Marie-Tooth/Neuropathy, Hereditary, With Liability To Pressure Palsies & 7 & 4 & 3 & - \\
\hline $182290 / 610883$ & Smith-Magenis/17p11.2 & 10 & 8 & 2 & - \\
\hline 613675 & $17 q 11.2$ & 4 & 4 & - & - \\
\hline 137920 & Renal Cysts And Diabetes & 10 & 10 & - & - \\
\hline $610443 / 613533$ & $17 q 21.31$ & 3 & 2 & 1 & - \\
\hline 115470 & Cat-Eye & 1 & - & $1 \wedge$ & - \\
\hline 188400/192430/608363 & $22 q 11.2$ & 83 & 64 & 19 & - \\
\hline 611867 & Distal 22q11.2 & 9 & 2 & 7 & - \\
\hline 606232 & Phelan-Mcdermid & 8 & 8 & - & - \\
\hline 308100 & X-linked Ichthyosis & 11 & $11 \wedge \wedge$ & - & - \\
\hline 312080 & Pelizaeus-Merzbacher & 1 & - & - & 1 \\
\hline $312750 / 300260$ & Rett/MECP2 & 2 & - & $2 \wedge \wedge \wedge$ & - \\
\hline
\end{tabular}

\$ Susceptibility locus / incomplete penetrance.

* Tatton-Brown (2009). 15q overgrowth syndrome: a newly recognized phenotype associated with overgrowth, learning difficulties, characteristic facial appearance, renal anomalies and increased dosage of distal chromosome 15q. Am J Med Genet 149:147.

** Hanner (2009). Recurrent reciprocal deletions and duplications of 16p13.11: the deletion is a risk factor for MR/MCA while the duplication may be a rare benign variant. J Med Genet 46:223.

$\wedge$ This patient was mosaic.

$\wedge \wedge$ all cases were $\mathrm{x} 0$ copy nullisomy in males.

$\wedge \wedge \wedge$ all cases were $x 2$ copies duplication in males.

For apparently de novo, non-LCR-mediated, interstitial imbalance, karyotype or FISH analysis of the parents is also necessary to exclude a balanced insertional translocation, the presence of which would carry an approximately $50 \%$ risk for the parents in future pregnancies.
Nowakowska et al. [11] report a frequency of approximately $2.1 \%$ of insertional translocation among families of patients with apparently de novo CNVs. In our experience, parental samples for chromosome rearrangement studies are rarely provided; we were able to 
complete FISH studies on only 12/226 (5\%) families with apparently de novo findings, and found one parental insertional translocation.

\section{Incidental findings}

These are unavoidable for any whole genome test and can be difficult to deal with clinically, especially with late onset conditions and cancer-susceptibility genes where little may be known of the prevalence or penetrance of clinical features associated with imbalances. In collaboration with our clinical oncology colleagues, we identified 58 genes, imbalance for which was considered likely, based on published studies, to confer increased risk of malignancy [12]. 80 patients in the cohort reported here had imbalance for one of these genes; these imbalances were reported with a recommendation for family studies and genetic counselling. In addition, we have chosen to highlight any other incidental findings where there is a possibility that they may have a significant impact on the health of a patient.

\section{Balanced rearrangements}

Despite the increase in resolution and the higher diagnostic yield associated with aCGH testing, there may be concern that without visualisation of chromosomes by traditional cytogenetic techniques, balanced rearrangements will not be detected. These rearrangements may disrupt gene function without causing any loss of coding material, and hence may be important diagnostically. However, the prevalence of de novo apparently balanced rearrangements associated with abnormal phenotype detected by G-banded chromosome analysis is very low, and some of these may in fact be unbalanced at the submicroscopic level; aCGH testing may reveal this imbalance without the need for karyotype analysis first. The increase in diagnostic yield by the use of aCGH remains of far greater patient benefit than the extremely small number of cases where a balanced rearrangement may disrupt an important gene. Whether in a state-funded or private service, the additional benefit of traditional banded chromosome analysis in addition to aCGH testing is likely to be extremely marginal.

\section{Summary}

This report describes 8,794 first line aCGH tests in a statefunded diagnostic laboratory. To our knowledge, this is the largest cohort of patients to date to be reported using this approach, and the results demonstrate that aCGH is a robust and cost-effective alternative to G-banded karyotype analysis, and provides a higher diagnostic detection rate. Implementation of a patient vs patient hybridisation strategy reduces costs, allowing more widespread testing, and therefore adding to the sum of information and understanding of the role of genomic imbalance in disease.
Experience of the challenges in interpretation and reporting of aCGH results will inform the implementation into clinical diagnostic service of higher resolution technologies such as whole exome and whole genome sequencing.

\section{Competing interests}

The authors declare that they have no competing interests.

\section{Authors' contributions}

JWA designed and constructed the clinical database and compiled the data. KM led the development of the custom MLPA follow-up service, RPH and AB helped with reporting pathways and SB supervised patient matching protocols. CMO designed the hybridisation strategies and led the diagnostic service. JWA and CMO wrote the paper. All authors contributed to interpretation of the array findings, participated in the diagnostic service, and reviewed the manuscript. All authors read and approved the final manuscript

\section{Acknowledgements}

We are grateful to our clinical colleagues for supporting this work and to the other members of the Cytogenetics Department for their enthusiastic participation

\section{Author details}

'Cytogenetics Department, Guy's and St Thomas' NHS Foundation Trust, London SE1 9RT, UK. ²Cytogenetics Department, GSTS Pathology, London SE1 9RT, UK.

Received: 14 January 2013 Accepted: 13 February 2013

Published: 5 April 2013

\section{References}

1. Miller DT, Adam MP, Aradhya S, Biesecker LG, Brothman AR, Carter NP, Church DM, Crolla JA, Eichler EE, Epstein CJ, et al: Consensus statement: chromosomal microarray is a first-tier clinical diagnostic test for individuals with developmental disabilities or congenital anomalies. Am J Hum Genet 2010, 86:749-764.

2. Park SJ, Jung EH, Ryu RS, Kang HW, Ko JM, Kim HJ, Cheon CK, Hwang SH, Kang HYCP: Clinical implementation of whole-genome array CGH as a first-tier test in 5080 pre and postnatal cases. Mol Cytogenet 2011, 4:12.

3. Ahn JW, Mann K, Walsh S, Shehab M, Hoang S, Docherty Z, Mohammed S, Mackie Ogilvie CCP: Validation and implementation of array comparative genomic hybridisation as a first line test in place of postnatal karyotyping for genome imbalance. Mol Cytogenet 2010, 3:9.

4. Hoang S, Ahn J, Mann K, Bint S, Mansour S, Homfray T, Mohammed S, Ogilvie CM: Detection of mosaicism for genome imbalance in a cohort of 3,042 clinical cases using an oligonucleotide array CGH platform. Eur J Med Genet 2011, 54:121-129.

5. lafrate AJ, Feuk L, Rivera MN, Listewnik ML, Donahoe PK, Qi Y, Scherer SW, Lee C: Detection of large-scale variation in the human genome. Nat Genet 2004, 36:949-951.

6. Hills A, Ahn JW, Donaghue C, Thomas H, Mann K, Ogilvie CM: MLPA for confirmation of array CGH results and determination of inheritance. Mol Cytogenet 2010, 3:19.

7. Mann K, Donaghue C, Fox SP, Docherty Z, Ogilvie CM: Strategies for the rapid prenatal diagnosis of chromosome aneuploidy. Eur J Hum Genet 2004, 12:907-915.

8. Vermeesch JR, Balikova I, Schrander-Stumpel C, Fryns JP, Devriendt K: The causality of de novo copy number variants is overestimated. Eur J Hum Genet 2011, 19:1112-1113.

9. Itsara A, Wu H, Smith JD, Nickerson DA, Romieu I, London SJ, Eichler EE: De novo rates and selection of large copy number variation. Genome Res 2010, 20:1469-1481.

10. van Bon BW, Mefford HC, Menten B, Koolen DA, Sharp AJ, Nillesen WM, Innis JW, de Ravel TJ, Mercer CL, Fichera M, et al: Further delineation of the $15 q 13$ microdeletion and duplication syndromes: a clinical spectrum varying from non-pathogenic to a severe outcome. J Med Genet 2009, 46:511-523 
11. Nowakowska BA, de Leeuw N, Ruivenkamp CA, Sikkema-Raddatz B, Crolla JA, Thoelen R, Koopmans M, den Hollander N, van Haeringen A, van der Kevie-Kersemaekers AM, et al: Parental insertional balanced translocations are an important cause of apparently de novo CNVs in patients with developmental anomalies. Eur J Hum Genet 2012, 20:166-170.

12. Pichert G, Mohammed SN, Ahn JW, Ogilvie CM, Izatt L: Unexpected findings in cancer predisposition genes detected by array comparative genomic hybridisation: what are the issues? J Med Genet 2011, 48:535-539.

doi:10.1186/1755-8166-6-16

Cite this article as: Ahn et al:: Array CGH as a first line diagnostic test in place of karyotyping for postnatal referrals - results from four years' clinical application for over 8,700 patients. Molecular Cytogenetics 2013 $6: 16$.

\section{Submit your next manuscript to BioMed Central and take full advantage of:}

- Convenient online submission

- Thorough peer review

- No space constraints or color figure charges

- Immediate publication on acceptance

- Inclusion in PubMed, CAS, Scopus and Google Scholar

- Research which is freely available for redistribution 